\title{
Magnus wind turbine: the effect of sandpaper surface roughness on cylinder blades
}

\begin{abstract}
The Magnus wind turbine is an invention that uses rotating cylinders as blades to extract energy from the wind. This invention overcomes the limitation of operating a wind turbine at low wind speed conditions. However, research regarding the torque generated by enhancing the surface roughness of the Magnus wind turbine is still lacking. Thus, the study aims to understand the effect of varied sandpaper surface roughnesses on the Magnus wind turbine torque output. The approaches used are: experimentation using a 6 cylinders model inside a wind tunnel for Magnus force comparison, a Magnus wind turbine model for torque performance and smoke flow visualisation for boundary layer analysis. The results show that the torque coefficient produced by P40 sandpaper to smooth the surface roughness is 0.079 0.016 , which is nearly a five times improvement in the torque coefficient. On the other hand, the tip speed ratio further increases from smooth to rough surface enhancement $(0.057-$ 0.147). This significant finding indicates that the Magnus wind turbine performance can be further improved using sandpaper surface roughness.
\end{abstract}

Keyword: Magnus wind turbine; Sandpaper surface roughness; Cylinder blades; Wind turbine 